\title{
Accelerated MR thermometry using the Kalman filter
}

\author{
Li Zhao ${ }^{1 *}$, Samuel Fielden ${ }^{1}$, Wilson Miller ${ }^{1}$, Xue Feng ${ }^{1}$, Max Wintermark ${ }^{1}$, Kim Butts Pauly $^{2}$, Craig Meyer ${ }^{1}$ \\ From Current and Future Applications of Focused Ultrasound 2014. 4th International Symposium \\ Washington, D.C, USA. 12-16 October 2014
}

\begin{abstract}
Background/introduction
Magnetic resonance (MR) imaging plays an important role in monitoring thermal treatment. It can quantify thermal dose with temperature maps based on the proton-resonance frequency shift. Volumetric coverage is desirable, but acquiring multiple slices imaging is time consuming. Therefore, accelerated methods are needed to improve the spatial and temporal resolution in MR thermometry. Multi-channel coils are not widely available for MR-guided FUS systems, so conventional parallel imaging methods cannot be used for acceleration. Compressed sensing methods show promise, but the computation is currently too slow to provide real-time feedback. The Kalman filter is an optimal estimation method that has been widely used for real-time tracking in other fields. It has been studied for filtering of temperature for FUS. Here we apply it to accelerate image acquisition for thermometry.
\end{abstract}

\section{Methods}

The Kalman filter (KF) uses prior state information to predict the current state with a dynamic system model:

$\mathrm{x}(\mathrm{k})=\mathrm{x}(\mathrm{k}-1)+\mathrm{w}(\mathrm{k}-1)$

$\mathrm{z}(\mathrm{k})=\mathrm{U}(\mathrm{k}) \mathrm{Fx}(\mathrm{k})+\mathrm{v}(\mathrm{k})$

$x(k)$ is the target image at the kth frame and the first function describes the state transition. $\mathrm{z}(\mathrm{k})$ is the corresponding acquired data. $\mathrm{F}$ is a Fourier transform operator and $\mathrm{U}(\mathrm{k})$ is an undersampling pattern. $\mathrm{w}$ and $\mathrm{v}$ are the system and measurement noise, assumed to have white Gaussian distributions with covariance matrices estimated by the KF. w models state changes resulting from heating. A numerical phantom was used to validate the proposed method. One normalized slice was sampled with a $128 \times 128$ matrix. The focal spot followed a 2D Gaussian distribution spatially. The temperature evolves with exponential increase and decay, with 15-degree peak. 100 image frames were simulated with complex Gaussian noise (std $=0.01)$. A gel phantom was tested with a HIFU system (RK-100, FUS Instruments Inc., Toronto) in a 3T Siemens Trio. Fully sampled data were acquired by a gradient echo sequence with $64 \times 64$ matrix, FOV $64 \mathrm{~mm} \times 64 \mathrm{~mm}$ and resolution $1 \mathrm{~mm} \times 1 \mathrm{~mm} \times 5 \mathrm{~mm}$. $\mathrm{TR} / \mathrm{TE}=15 / 6 \mathrm{~ms}$ and bandwidth $500 \mathrm{~Hz}$ provided temporal resolution $0.96 \mathrm{~s}$ per frame. The sequence acquired data continuously during three consecutive 30 -second intervals corresponding to baseline, continuous sonication, and cooling. Data were undersampled by a factor of 2 along the phase encoding direction (y) and reconstructed by zero filling, view sharing, KF, and KF with first frame initialized by view sharing. Temperature maps were calculated by the PSF method. The temperature map of fully sampled k-space was chosen as the standard to evaluate the performance of the above methods.

\section{Results and conclusions}

Figs. 1 and 2 show the simulated spatial and temporal temperature maps. The KF method produced negligible aliasing artifacts in the temperature map (top) and resulted in better approximation of the standard temperature with less error (bottom). With the first image initialized by view sharing, KF further reduced the error in the first few frames. Fig. 3 shows the experimental temporal temperature maps. Fig. 4 shows the temporal profile of the focal spot ( $3 \times 3$ pixels). The KF method approximated the fully sampled image accurately and provided a temperature map with less error. In conclusion, the KF method can estimate temperature accurately with a speed-up of at least $2 \mathrm{X}$, enabling real-time thermometry with greater spatial coverage.

'University of Virginia, Charlottesville, Virginia, United States

Full list of author information is available at the end of the article 

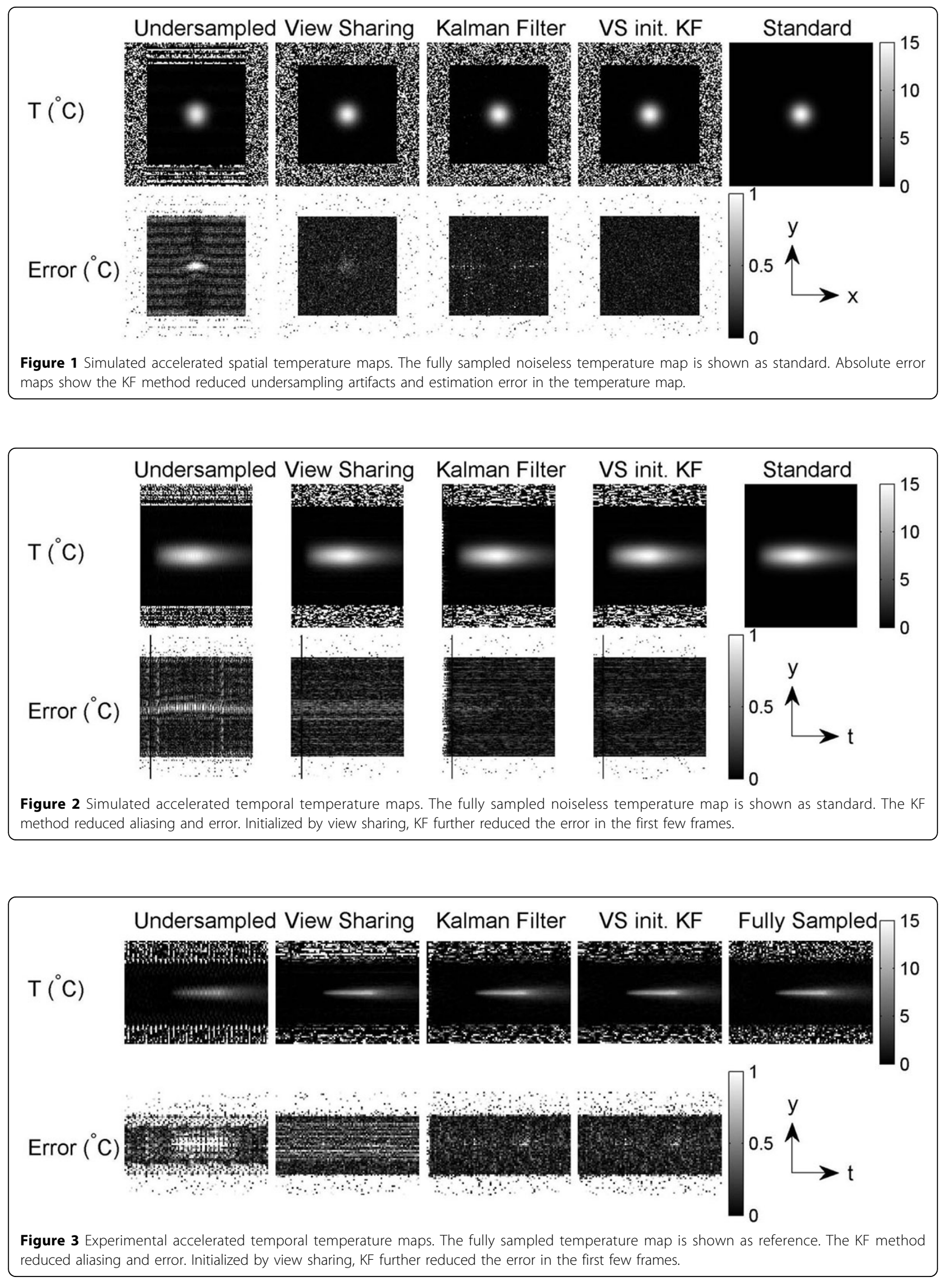


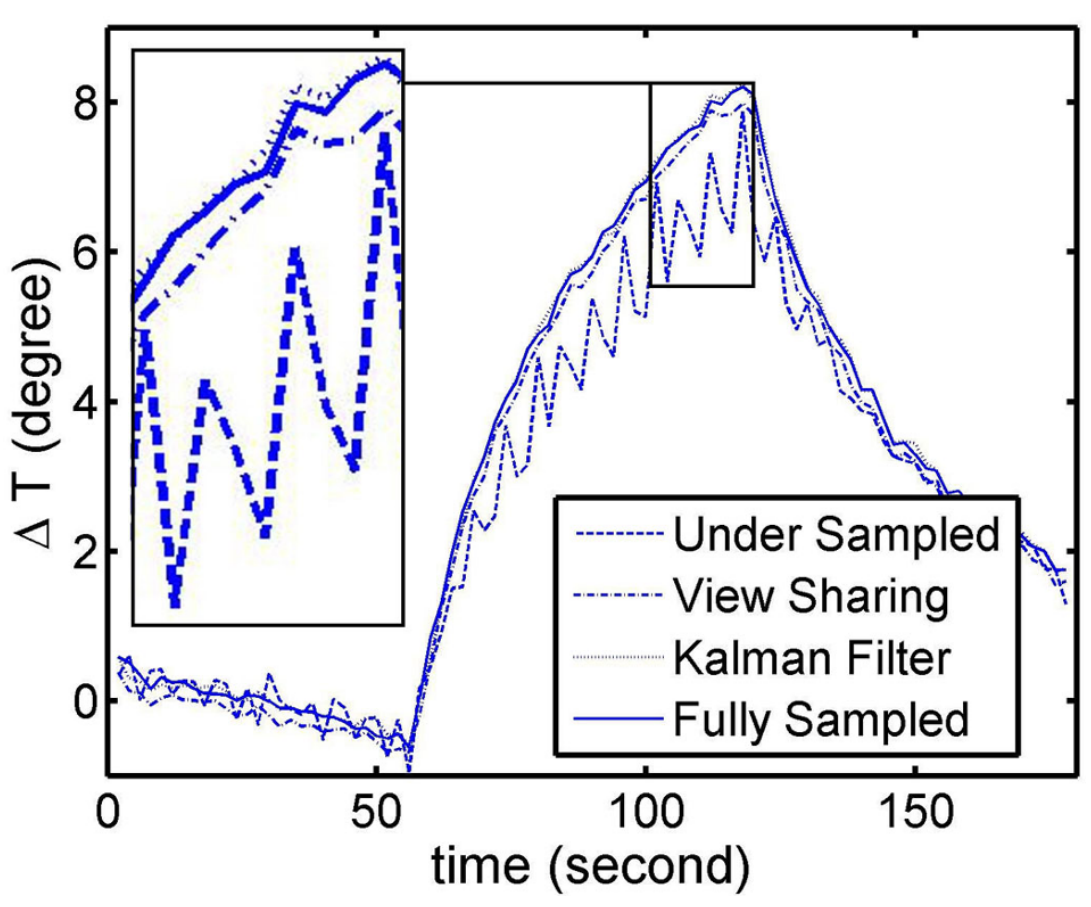

Figure 4 Temporal plot of focal spot in gel phantom. ROls ( $3 \times 3$ pixels) were selected around the highest temperature. The average temperature shows the KF method approximated the temperature more accurately than other methods with $2 \mathrm{X}$ acceleration.

Acknowledgements (Funding)

Focused Ultrasound Foundation, Siemens Medical Solutions.

\section{Authors' details}

'University of Virginia, Charlottesville, Virginia, United States. ${ }^{2}$ Stanford

University, Stanford, California, United States.

Published: 30 June 2015

doi:10.1186/2050-5736-3-S1-P40

Cite this article as: Zhao et al:: Accelerated MR thermometry using the

Kalman filter. Journal of Therapeutic Ultrasound 2015 3(Suppl 1):P40.

Submit your next manuscript to BioMed Central and take full advantage of:

- Convenient online submission

- Thorough peer review

- No space constraints or color figure charges

- Immediate publication on acceptance

- Inclusion in PubMed, CAS, Scopus and Google Scholar

- Research which is freely available for redistribution 